\title{
Nature of Contacts in Centric Occlusion in 32 Adults
}

\author{
J. RUSSELL ANDERSON, JR. and GEORGE E. MYERS \\ Crown and Bridge Department, University of Michigan School of Dentistry, Ann Arbor, \\ Michigan 48104, USA
}

An evaluation of the location and nature of occlusal contacts in centric occlusion of 32 adults revealed teeth that had no occlusal contact, no opposing tooth, contact on flat surfaces, contact on one or more inclined planes, and contacts on a combination of flat and inclined planes.

Understanding the nature of the intercuspation of the teeth in centric occlusion is necessary for the practice of restorative dentistry. Intercuspation of the teeth is one of the factors responsible for maintaining the correct position of the teeth. Changes in occlusal anatomy at the time restorations are made may result in movement of the involved teeth to new positions of equilibrium. The ideal patterns of tooth intercuspation have been described by Friel $^{1,2}$ for deciduous, mixed, and adult dentitions. Friel described the location of the various contacts between the maxillary and mandibular teeth and divided the contacts into four categories: (1) cusp point contact with a fossa, a groove, or the occlusal embrasure between teeth, (2) ridge contact with an embrasure, (3) ridge contact with a groove, and (4) surface contacts. Friel's illustrations have become classics in this field (Fig 1). More recently, Ramfjord and $\mathrm{Ash}^{3}$ have published drawings of ideal tooth contacts in centric occlusion. In general, these ideal occlusal relations are of such a nature that the posterior mandibular and maxillary teeth are locked when in centric occlusion and mutually support each other against other forces acting on them.

Hellman, ${ }^{4}$ in a discussion of the factors that influence occlusion, classified contacts into the same four groups as Friel. Stuart ${ }^{5}$

This paper is based on data collected in a study supported by USPHS Grant DE-02170-01A1.

Received for publication June 12, 1969. called the fossae of teeth "stamp mills" and the cusps "pestles" and classified occlusal contacts as "tooth to tooth" occlusion, "tooth to two tooth" occlusion, and "cusp to fossa" occlusion.

Little evidence has been presented in the literature about the location and nature of tooth contacts as they exist in the mouths of typical clinical patients. Petit ${ }^{6}$ developed a method to record permanently the extent of occlusal contacts. Thin wax bites were used and recorded in photographs. However, no attempt was made to classify the location of these contact areas on each individual tooth in relation to cuspal inclines, marginal ridges, cusp tips, or fossae.

The daily experience of any clinician reveals that occlusal contacts often deviate from the ideal. This paper presents data collected from 32 adults regarding the location and nature of the contacts in centric occlusion between the maxillary and mandibular teeth. The data are from a serial study of occlusion in adults that covered a much wider field than is reported here.

\section{Materials and Methods}

Thirty-two patients, aged 20 to 59 years, were included in this study. All patients had complete dentitions (except for some missing third molars) or dentitions that could be restored with a fixed prosthesis. Most patients had relatively few restorations in their teeth (less than ten functional interproximal restorations). All patients had practiced oral hygiene, and none exhibited any pathologic periodontal conditions. No abnormal wear facets were present. A set of complete mouth impressions was taken for each of the 32 patients with agar hydrocolloid impression material and the impressions were poured in improved stone.

The contact areas of the teeth in the maximum intercuspation position (centric 


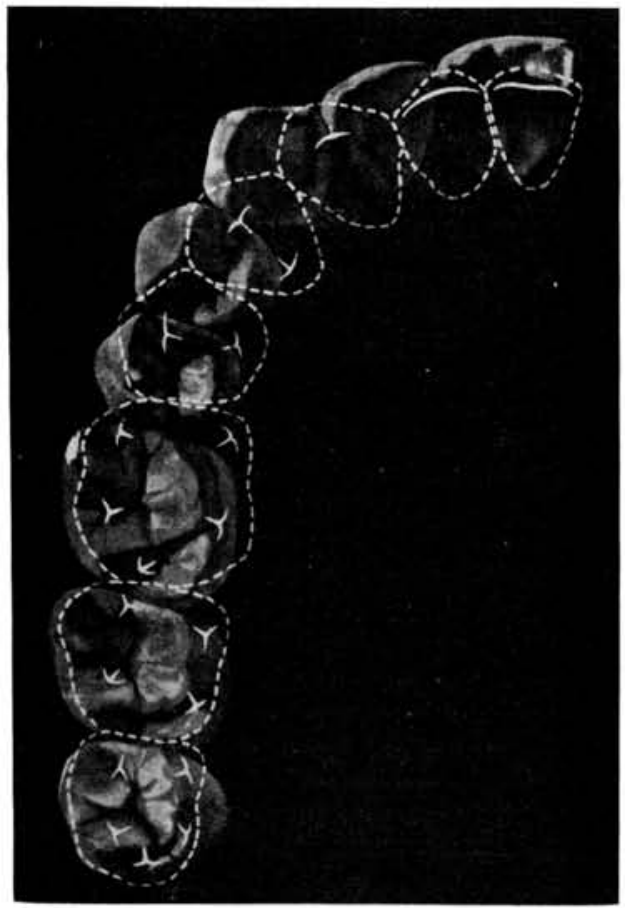

FIg 1.-Ideal occlusion shows maxillary teeth with outlines of opposing mandibular teeth. ${ }^{1}$ (Reprinted with permission of publisher.)

occlusion) were determined by use of 28 gauge green casting wax* at room temperature, with the patient's teeth in that occlusal position. The wax was pressed thin or cut through in the areas of occlusal contact.

Three distinct types of indentations were noted on the wax wafer: an indentation with little or no thinning of the wax; an indentation with thinning of the wax readily recognized by the formation of a translucent area that was easily seen by holding the wafer in front of a light source; and perforation of the wafer. The first type of indentation represents near contact, the second type represents contact, and the third type represents supracontact. In this study, the latter two types of indentation were recorded as contacts.

Then, in similar fashion, the centric occlusion contacts of the teeth on the casts were determined by interposing a new piece of wax between the occlusal surfaces of the

* Kerr Dental Manufacturing Co., Detroit, Mich. teeth, approximating the casts in the maximum intercuspation position by hand, and applying pressure. The wax was pressed thin or cut in the areas of contact in centric occlusion when the wax registration was removed. Red butterfly ribbon was interposed between the teeth of the casts and pressure was applied to the casts in centric occlusion. The areas of contact in centric occlusion were marked in red on the teeth of the casts. These red markings acted as a visual aid in interpreting the wax registrations by making it easier to transfer or record the occlusal contact areas onto charts in the subsequent collection of data (Fig 2).

The indentations and cut areas on the wax that were taken from the patient's mouth were carefully compared visually with those indentations and cut areas on the wax that were obtained from the approximation of the casts by hand. These areas were found to coincide in location (Fig 3).

By comparison of the oral and cast wax recordings and by replacing the wax recordings on the casts, it was possible to record accurately the locations of the centric occlusion contact areas of the maxillary or mandibular teeth on charts made from photographs of dental casts (Fig 4). The areas of contact as recorded on the charts were then checked with those areas

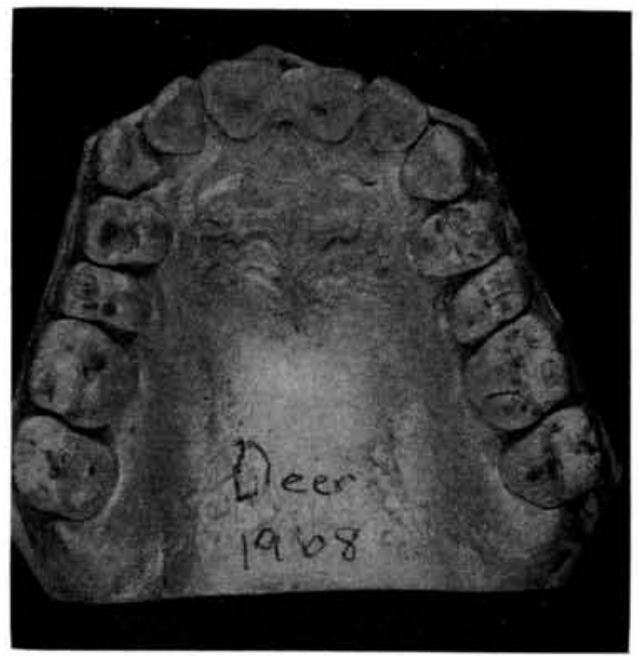

FIG 2.-Maxillary cast shows butterfly ribbon markings, indicating centric occlusal contacts. 


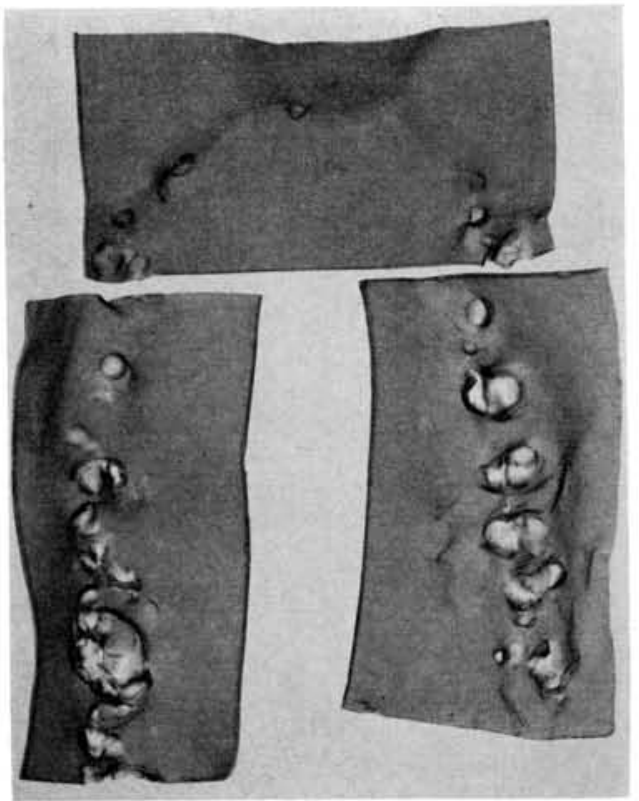

FIG 3.-Indentations in 28 gauge green wax shows near contacts; cut areas indicate supracontact in centric occlusion.

marked on the casts by the butterfly ribbon. Visual examination of the approximated casts gave further verification of the location on the teeth of the contacts in centric occlusion.

The occlusal relation for each individual tooth was classified and recorded as one of the following six types:

1. No opposing teeth:

2. No contact with an opposing tooth;

3. Contact with a "flat" plane; eg, the bottom of a fossa, a marginal ridge, or a cusp tip;

4. Contact on an inclined plane; eg, a cuspal incline or lingual or labial surface of an incisor;

5. Contact with two or more converging inclined planes; eg, planes on each side of a fossa:

6. Combination of a flat and inclined plane; eg, any combination of 3,4 . or 5 .

\section{Results}

The 32 patients examined in this study had a total of 947 teeth. There were 565 posterior teeth and 382 anterior teeth. Eight patients had occlusions in which all the teeth made contact in centric occlusion.
The remaining 24 patients had from 1 to 12 teeth that did not contact in centric occlusion. The anterior teeth had narrow incisal edges, whereas the posterior teeth had broad occlusal surfaces. This difference in morphology created a difference in the nature of occlusal contacts of the anterior teeth and of the posterior teeth. It was convenient, therefore, to examine the data in relation to anterior and posterior teeth separately.

ANTERIOR TEETH.-Of the 382 anterior teeth, 72 did not contact an opposing tooth in centric occlusion. A mandibular incisor in each of two patients had no contact because the maxillary antagonist was missing (type 1). Contact could be reestablished by placing a maxillary, three unit, fixed bridge. Seventy anterior teeth had no contact in centric occlusion even though the opposing tooth was present (type 2). One hundred fifty-four maxillary teeth made contact in centric occlusion with 156 mandibular teeth. In all instances, the labial margin of the incisal edge of the mandibular tooth contacted the lingual surface of the maxillary teeth. All these contacts were, therefore, in the single incline plane (type 4).

Posterior TEeTH.-The data collected concerning the 565 posterior teeth showed that 12 posterior teeth had no contact with an opposing tooth because the opposing tooth was missing (type 1). Nine of these 12 posterior teeth were third molars. In the remaining three posterior teeth, three mandibular molars, occlusal contact could be achieved again by placing fixed bridges. Twelve posterior teeth had no contact with an opposing tooth even though the opposing tooth was present in the dental arch (type 2). Most of these teeth were third molars.

Thirty-seven posterior teeth had centric occlusion contacts on flat surfaces only (type 3 ). Six posterior teeth exhibited end to end or cusp tip to cusp tip contact in centric occlusion. Two patients each had one pair of second molars with this type of occlusal contact, and the remaining two teeth were a maxillary third molar and maxillary second premolar. Thirty-one posterior teeth had centric occlusion contacts in which a cusp tip occluded with the lowest point of the marginal ridge of the op- 

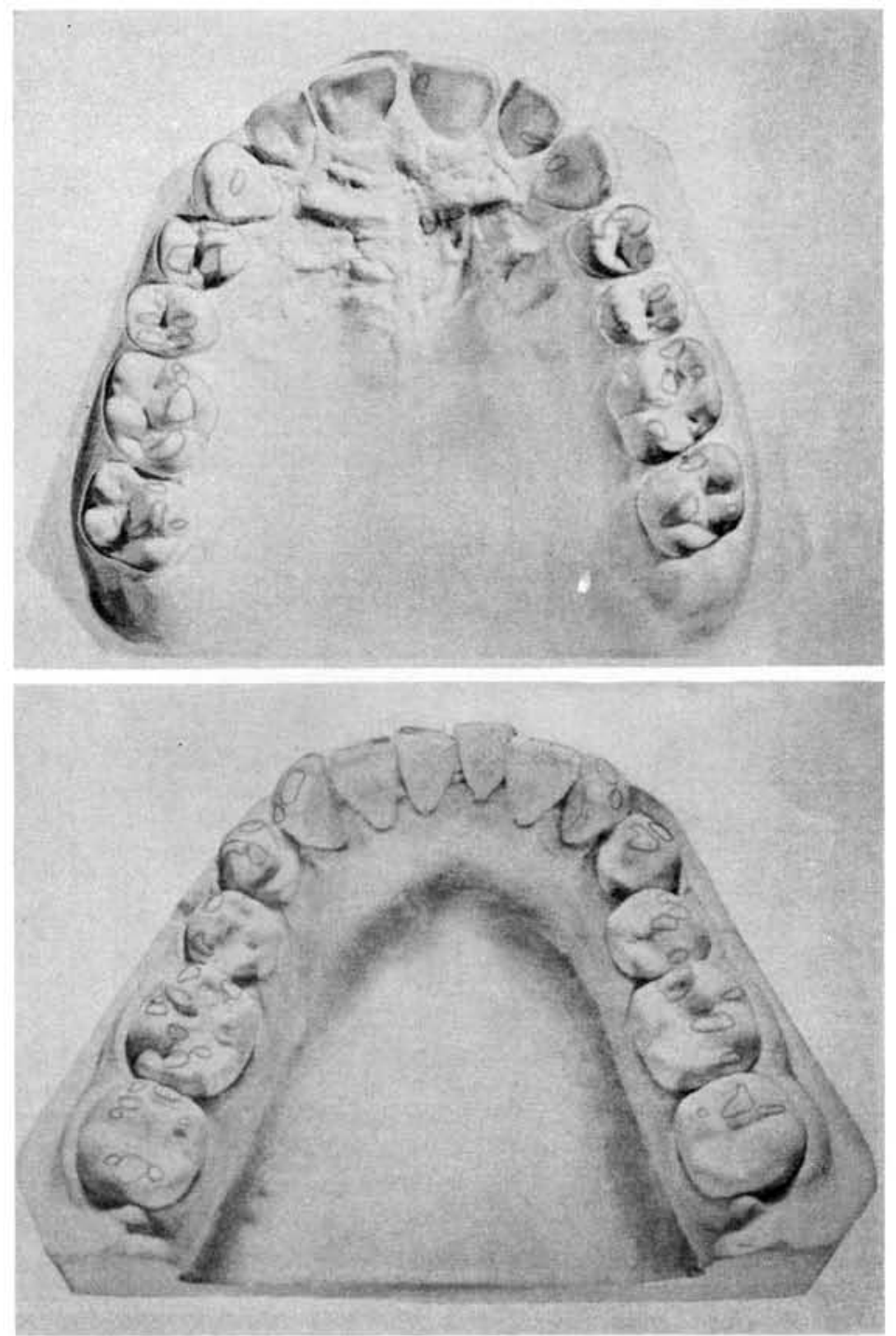

FIG 4.-Centric occlusal contacts recorded in pencil on charts of maxillary and mandibular teeth.

posing tooth (type 3 ). Sixteen teeth found in the posterior region of the mouth had contact in centric occlusion on one inclined plane only (type 4).

One hundred fifty-three posterior teeth had centric occlusion contact areas on two cuspal inclines only and did not contact any flat surfaces (type 5). The majority of the posterior teeth (335) had contact areas in a combination of places, ie, on cuspal inclines and flat surfaces, such as a fossa or marginal ridge, at the same time (type 6).
The distribution of the six types of occlusal contacts among the 32 patients is shown in Tables 1 and 2.

\section{Discussion}

No patient had an ideal occlusion as described by Friel. ${ }^{1,2}$ The significant deviations from the ideal, may have been caused by a variety of factors, such as placement of restorations, attrition, continuous eruption, occlusal habits, early loss of deciduous teeth, disturbances in the eruption sequence, 


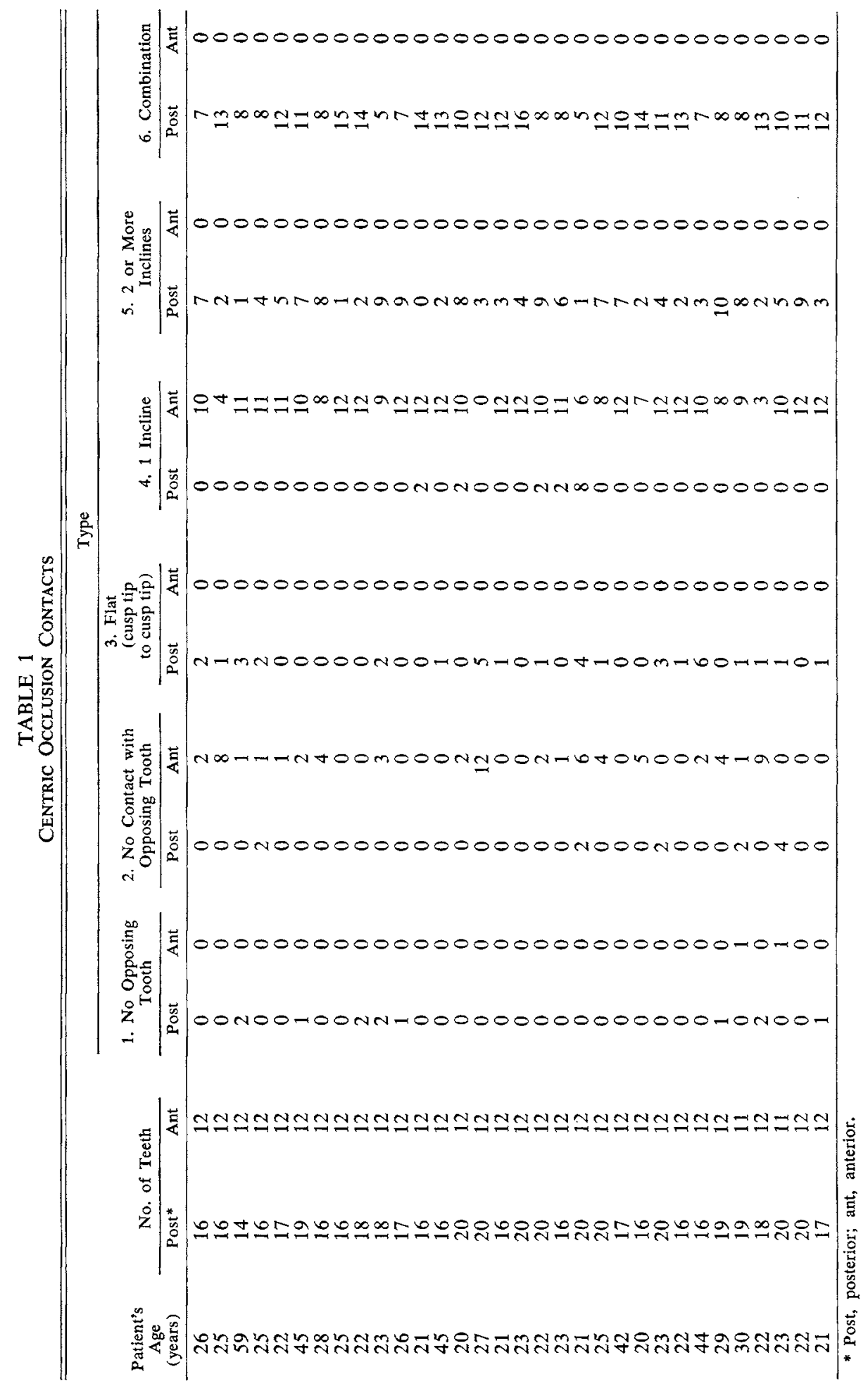


TABLE 2

Frequency of Types of Centric OCclusion Contacts

\begin{tabular}{lrrrrr}
\hline \multirow{2}{*}{ Type } & \multicolumn{2}{c}{ Frequency } & & \multicolumn{2}{c}{ Range } \\
\cline { 2 - 3 } \cline { 5 - 6 } & Ant* & Post & & Ant & Post \\
\hline 1. No opposing tooth & 2 & 12 & & $0-1$ & $1-2$ \\
2. No contact with & & & & $1-12$ & $2-4$ \\
opposing tooth & 70 & 12 & & 0 & $1-6$ \\
3. Flat (cusp tip to cusp tip) & 0 & 37 & & $0-12$ & $2-8$ \\
4. 1 incline & 310 & 16 & & 0 & $0-10$ \\
5. 2 or more inclines & 0 & 153 & & 0 & $5-16$ \\
6. Combination & 0 & 335 & & &
\end{tabular}

loss of the permanent teeth, periodontal disease, and orthodontic problems.

Occlusal relations in which the opposing tooth is missing (type 1) are frequently seen clinically. The expectation in these situations is that the unopposed tooth will continue to erupt, the teeth adjacent to the edentulous region will drift, and the rest of the classical effects of tooth loss on the dental arch will occur, unless an antagonist is provided by a prosthetic appliance. The unopposed tooth may be extracted in certain instances, as is often done with an unopposed third molar.

Seventy anterior teeth and 12 posterior teeth had no contact with the opposing tooth (type 2). The absence of occlusal contact between the anterior teeth is well recognized clinically and, when present, is regarded as normal and stable, although no serial data have been published to substantiate this. Beyron, ${ }^{7}$ in a study of 46 Australian aboriginal adults, found "a minute space between the upper and lower incisors of most of the subjects." The situation of the posterior teeth exhibiting type 2 occlusal contacts is not clear. Most of these third molars in patients under 25 years of age may still be in the state of active eruption.

Three-hundred-ten anterior teeth and 16 posterior teeth had occlusal contacts on a single inclined plane (type 4). For anterior teeth, this situation is clinically considered normal and stable. In anterior and posterior teeth with type 4 occlusal contact areas, there must be an equilibrium in the physiologic forces of the tongue, lips, cheeks, and occlusion that forces the teeth to retain their relative position. Posterior teeth with occlusal contacts on a single inclined plane are also dependent on the same equilibrium of the physiologic forces for their positional stability. It is conceivable that through continuous eruption or a change in the oral forces, posterior teeth may slide over the single incline planes to a new position, thus establishing a definite occlusal stop or a "lock." However, only serial study will reveal whether these positions are stable and whether the teeth are, in fact, in equilibrium with the forces acting on them or whether the resulting forces perhaps change from time to time so that some variation in position of these teeth may be expected.

The 37 posterior teeth that exhibited occlusal contact on flat surfaces (type 3 ) also depend on the same equilibrium of the occlusal and oral forces for their maintenance of the existing contact, as do the teeth with type 4 occlusal contact areas. The six teeth with end to end or cusp tip to cusp tip contact are influenced even more by the physiologic forces for the maintenance of the present occlusal contact than those teeth with cusp tip to marginal ridge contact. The teeth with contact types 5 and 6 are all posterior teeth. They are locked in place by existing cuspal inclines and do not depend on the existence of an equilibrium in the physiologic forces of the lips, tongue, and cheeks for their positional stability to the same extent as the teeth with contact types 1 through 4 . However, interproximal and occlusal attrition and the placement of restorations affect the occlusal contact area relationships of these teeth to a greater extent than those teeth that have contact area types 1 through 4 .

Of the several types of tooth contacts, types 1 through 4 do not lock the teeth into a stable positional relationship, and the teeth are subject to the influence of the 
forces from the oral musculature. Contact types 5 and 6 tend to lock the teeth into a stable position that is probably secure against the influence of the tongue, cheeks, and other functional forces. Whether any of the 947 teeth that had the described centric occlusion contact areas are stable in position and contact area depends on an equilibrium of all the forces that act on them. Only a serial examination over a period of time will reveal whether these teeth are stable or are moving to new positions.

\section{Conclusions}

Long-term serial studies are needed to determine the positional stability of the occlusal contact areas. The "ideal occlusion" was not found in any of the teeth examined. Most of the teeth that had no occlusal contact with an opposing tooth were anterior teeth. The anterior teeth with occlusal contacts occluded on inclined planes. Most posterior teeth occluded on two or more inclined planes leading to a fossa or on a combination of inclined and flat planes. Relatively few teeth in this study exhibited cusp tip to cusp tip or cusp tip to marginal ridge occlusion.

The authors acknowledge the aid of $O$. C. Applegate, who developed the photographs used in Figure 4.

\section{References}

1. Friel, S.: Occlusion, Observations on Its Development from Infancy to Old Age, Int J Orthodont 13:323-343, 1927.

2. FrIEL, S.: The Development of Ideal Occlusion of the Gum Pads and the Teeth, J Orthodont 40:196-227, 1940.

3. RAMFJORD, S.P., and Ash, M.M.: Occlusion, Philadelphia: W. B. Saunders Co., 1966.

4. Hellman, M.: Factors Influencing Occlusion, Angle Orthodont 12:3-27, 1942.

5. Stuart, C.E.: Good Occlusion for Natural Teeth, J Prosth Dent 14:716-724, 1964.

6. Petit, H.: The Occlusogram, the Objectivization of Occlusal Disorders, Actualités Odontostomat 77:37-59, 1967.

7. BeYron, H.L.: Occlusal Relations and Mastication in Australian Aborigines, Acta Odont Scand 22:597-678, 1964. 